\title{
Upregulation of CRMP4, a new prostate cancer metastasis suppressor gene, inhibits tumor growth in a nude mouse intratibial injection model
}

\author{
WEI ZHOU ${ }^{1 *}$, PEIGEN XIE $^{1 *}$, MAO PANG $^{1 *}$, BU YANG $^{1}$, YOUQIANG FANG ${ }^{2}$, TAO SHU $^{1}$, \\ CHANG LIU ${ }^{1}$, XUAN WANG ${ }^{1}$, LIANGMING ZHANG ${ }^{1}$, SHANGFU LI ${ }^{1}$ and LIMIN RONG ${ }^{1}$ \\ Departments of ${ }^{1}$ Spine Surgery and ${ }^{2}$ Urinary Surgery, The Third Affiliated Hospital \\ of Sun Yat-sen University, Guangzhou, Guangdong, P.R. China
}

Received August 9, 2014; Accepted September 23, 2014

DOI: 10.3892/ijo.2014.2705

\begin{abstract}
Prostate cancer, the most commonly diagnosed male cancer in North America, has a high incidence of bone metastasis. Our previous study showed collapsin response mediator protein 4 (CRMP4) gene inhibited prostate cancer migration and invasion. In this study, we investigated whether overexpression of CRMP4 gene in prostate cancer cells inhibit tumor bone metastasis. The stable prostate cancer cells overexpressing the CRMP4 gene were constructed using lentivirus infection. Prostate cancer bone metastasis nude mouse model was built though orthotopic prostate implantation, intracardiac injection and intratibial injection with CRMP4 overexpress and control cancer cells. Small animal PET/CT scanning results showed no difference of bone metastatic capacity in orthotopic and intracardiac injection models between CRMP4 overexpression and control group, while CRMP4 overexpression inhibited tumor growth in the intratibial injection model. Moreover, our in vitro study showed CRMP4 overexpression downregulates the Neuropilin1 (NRP1) expression and upregulate the Noggin expression. Immunohistochemical staining of the hind limbs of intratibial injection model was confirmed with cytological experiments. Taken together, our research indicated CRMP4 inhibits prostate cancer cells growth in the nude mouse bone microenvironment and this effect may relate with regulation of NRP1 and Noggin expression.
\end{abstract}

\section{Introduction}

Collapsin response mediator proteins (CRMPs) include five family members (CRMP1-5) and all family members have

Correspondence to: Professor Limin Rong, Department of Spine Surgery, The Third Affiliated Hospital of Sun Yat-sen University, 600 Tianhe Road, Guangzhou 510630, P.R. China

E-mail: ronglm21@163.com

*Contributed equally

Key words: collapsin response mediator protein 4, prostate cancer, bone metastasis, Noggin, Neuropilin1
$50-70 \%$ sequence homology with each other (1). CRMP is a cytosolic phosphoprotein and none of CRMP isoforms demonstrate any enzymatic activity $(2,3)$. Previous studies have shown that CRMP in Sema3A (collapsin-1) signaling, mediate axonal guidance and neuronal differentiation $(3,4)$. The downstream effectors of CRMP signaling is still not clear (1), research showed CRMP mediates microtubule dynamics and endocytosis (5-7). CRMP is mainly regulated by semaphorins and it has been shown that CRMP also combine with a variety of other signaling molecules (8-11) and this imply CRMP plays a role in numerous cellular processes.

Most studies of CRMP focus on its role in axonal guidance in the developing nervous system and CRMP has been show highly expressed in the developing and injured nervous system (1), however, recent study indicated that CRMP is involved in several malignant diseases (12). Shih et al reported that CRMP1 negatively correlated with lung cancer invasiveness (13), further study indicated LCRMP1, a long form isoform of CRMP1, promotes lung cancer cell invasiveness and this effect may relate to LCRMP1-WAVE1 combination and GSK-3 $\beta$ phosphorylation of LCRMP1 $(14,15)$. Both GSK-3 $\beta$ and WAVE-1 were involved in CRMP mediated axon outgrowth, indicating CRMP has diverse functions in different cellular processes. Other CRMP family members involved in cancer include CRMP2, CRMP4 and CRMP5, all were reported associated with different cancer cell migration, invasion, differentiation and clinical outcome in certain tumor types (16-19). In our previous study (20), we first identified CRMP4 as a prostate cancer metastasis suppressor factor by proteomics approach and verified that CRMP4 suppress prostate cancer cell line proliferation and invasion in vitro and the downregulation of CRMP4 in metastatic tumor may be due to the methylation of the $\mathrm{CpG}$ island within the promoter region of the CRMP4 gene.

Cancer metastasis is the end product of tumor genesis and development. Disseminated tumor cells arrest at target organ though vasculature, finally forming macroscopic neoplastic growth (21). Tumor metastasis is a highly inefficient process, extremely small percentage of tumor cells that enter into the systemic circulation ultimately develop into macroscopic metastasis $(22,23)$. Unlike lung cancer and breast cancer, which 
also have a high incidence of bone metastasis and often form osteolytic damage, most prostate cancer formed osteoblastic bone metastasis (24). Inefficient growth at the secondary site and high incidence of osteoblastic bone metastasis of prostate cancer indicate the specific tumor cell-organ interactions with host organ have a significant influence on the development of metastasis. During the process of bone metastasis, cytokines play an important role in the complex reciprocal interactions between the tumor cells and the bone microenvironment, factors secreted by tumor cells and bone stromal cells, which may contribute to the progress of metastatic tumor growth (25).

In our present study, we sought to investigate the role of CRMP4 in prostate cancer bone metastasis and the relationship of CRMP4 and the cytokines and proteins which correlate with prostate cancer bone metastasis.

\section{Materials and methods}

Cell culture. PC3 and DU145 cell lines were obtained from the ATCC (Rockville, MD, USA) and maintained in culture PRMI-1640 medium (Gibco, USA) supplemented with $10 \%$ fetal bovine serum (FBS, Gibco, USA). Cells were cultured in a humidified atmosphere at $5 \% \mathrm{CO}_{2}$ and $37^{\circ} \mathrm{C}$.

Lentiviral transduction. GFP-CRMP4 ${ }^{+}$lentivirus and a GFP lentivirus (Junhui Biology, China) were transfected into PC3 and DU145 cells in the presence of $5 \mu \mathrm{g} / \mathrm{ml}$ polybrene (Sigma, USA). Transduced cells were selected by FACS (fluorescenceactivated cell sorting, BD influx, USA) by GFP expression after transduced prostate cancer cells reached a total score of $10^{7}$. Western blot analysis, FCM (flow cytometer, BD LSRII, USA) and quantitative real-time PCR (qRT-PCR) were used to determine the effects of CRMP4 gene overexpression.

In vivo tumor models (animal experiments). The 4-5-week old male athymic nude mice (Vital River ${ }^{\circledR}$, Beijing, China) were studied to evaluate the in vivo metastatic behavior of tumor cells. All the experiments were approved by the Institutional Animal Care and Use Committee (approval no: XYXK-20120081) of Sun Yat-sen University.

Orthotopic implantation. Two groups of 7 animals each were used. Mice were anesthetized with xylazine (FaMu Chemical Plant, Nanjing, China) and ketamine (Fujian GuTian Pharma Co., China) though intraperitoneal injection. A lower middle abdominal incision was made and seminal vesicle and bladder were exposed to identify the mouse prostate. CRMP4 ${ }^{+} \mathrm{PC} 3$ cells $\left(10^{4} / 10 \mu \mathrm{l}\right)$ (CRMP4 overexpressing PC3 cells) and control PC3 were injected into the capsule of the prostate of the animals. The incision in the abdominal wall was closed with a 4-0 surgical suture. Baytril (2.5 $\mu \mathrm{g})$ (Bayer, Germany) was subcutaneously injected three days after operation to prevent infection. Small animal micro-PET/CT scan (Inveon, Siemens, Germany) was used to detect the skeletal metastasis 40 days after infection. fluorine-18 fluorodeoxyglucose $\left({ }^{18} \mathrm{~F}-\mathrm{FDG}\right)$ was used as a probe, $70 \mu \mathrm{Ci}{ }^{18} \mathrm{~F}$-FDG for each mouse and injected intravenously in conscious animals via the tail vein. Twenty-five minutes later, the mice were anesthetized and the scanning was performed $30 \mathrm{~min}$ after injection. The animals were placed on a heating pad to maintain body temperature throughout the procedure and visually monitored for breathing and any other signs of distress throughout the entire imaging period. Imagines were analysis by Inevon Research Workplace 4.1 software (Siemens, Germany). After the examination the animals were sacrificed and dissected to identify metastasis tumor tissue. Suspicious bone metastatic was fixed in $10 \%$ formalin for histological examination.

Intracardiac injection. Two groups of 7 animals each were used, The CRMP4 ${ }^{+} \mathrm{PC} 3$ and PC3 cells were injected into the left ventricle at a concentration of $10^{5} / 50 \mu \mathrm{l}$ of phosphatebuffered saline (PBS). Injection methods as previously described (26). Small animal micro-PET/CT scan and tissue processing was as indicated above.

Intratibial injection. Two groups of 7 animals each were used, the CRMP4 ${ }^{+} \mathrm{PC} 3$ and PC3 cells were injected into the left tibia medullary cavity of the two groups at a concentration of $10^{4} / 10 \mu 1$ of $5 \mu 1 \mathrm{PBS}$ and $5 \mu 1$ Matrigel (BD, USA). A $21-\mathrm{G}$ syringe was used to drill a hole though the tuberosity of tibia and cells were injected though $29-\mathrm{G}$ insulin syringe. Injection was done very slowly to prevent cells entering the soft tissue. No incision was made. The mice were sacrificed 40 days after injection and the left legs were harvested, $10 \%$ formalin-fixed and a high-resolution micro-CT (Inveon, Siemens, Germany) were used to measure bone destruction. CT imagines were scored as follows: 0 , normal; 1 , lytic lesion present within the medullary canal only; 2 , obliteration of one cortex; 3 , obliteration of two cortices. Two experienced orthopedists in a blinded manner identified the scores.

Quantitative real-time PCR ( $q R T-P C R$ ) analysis. The total RNA was isolated using TRIzol Reagent (Invitrogen, USA), cDNA synthesized with a Revert Aid first-strand cDNA synthesis kit (Takara, China). SYBR Premix Ex Taq (Life Technologies, USA) was used for qRT-PCR. Primers are listed in Table I. qRT-PCR analysis was performed on ABI 7500 Sequence Detection System (Applied Biosystems, USA). Gene expression relative to housekeeping gene GAPDH was calculated using the $2^{-\Delta \Delta \mathrm{Ct}}$ method. $2^{-\Delta \Delta \mathrm{Ct}}>2$ or $<1 / 2$ was considered statistically significant.

Western blot analysis. Total cellular proteins were extracted with RIPA lysis buffer kit (KeyGen Biotech, China). Samples were separated by $10 \%$ sodium dodecylsulfate polyacrylamide gel electrophoresis (SDS-PAGE) and then transferred to a polyvinylidene fluoride (PVDF) membrane. The CRMP4 and Noggin was assessed using primary rabbit anti-CRMP4 and rabbit anti-Noggin monoclonal antibody (Abcam, USA). NRP1 was assessed using primary rabbit anti-NRP1 monoclonal antibody (ABclonal, Cambridge). The membranes were incubated with primary antibodies and then horseradish peroxidase (HRP) secondary antibodies. An enhanced chemiluminescence reagent (ECL) kit (Millipore, USA) was used to detect the labeled proteins. GAPDH (rabbit anti-GAPDH monoclonal antibody, Jetway, China) was used to control equal loading quantity.

Histological experiments. Tissue specimens were decalcificated in $14 \%$ EDTA and embedded in paraffin, 5- $\mu \mathrm{m}$ sections 
Table I. Primer sequences.

\begin{tabular}{lll}
\hline Gene & \multicolumn{1}{c}{ Forward primer } & \multicolumn{1}{c}{ Reverse primer } \\
\hline Noggin & CCATGCCGAGCGAGATCAAA & TCGGAAATGATGGGGTACTGG \\
BMP2 & TGCGTACTCACGGTGGAATC & GTAAAACCCGTCTGTAGCTTCTT \\
BMP4 & ATGATTCCTGGTAACCGAATGC & CCCCGTCTCAGGTATCAAACT \\
BMP6 & AGCGACACCACAAAGAGTTCA & GCTGATGCTCCTGTAAGACTTGA \\
BMP7 & TCGGCACCCATGTTCATGC & GAGGAAATGGCTATCTTGCAGG \\
Sema3A & ACACCAGAAGAGATGAATGC & GCGTACAAGTGAGTCTGATT \\
NRP1 & GGCGCTTTTCGCAACGATAAA & TCGCATTTTTCACTTGGGTGAT \\
NRP2 & GCTGGCTATA-TCACCTCTCCC & TCTCGATTTCAAAGTGAGGGTTG \\
VEGFA & CTTTTCTCTGCCTCCACAATG & GAGTGGTTGACCTTCCTCCA \\
VEGFB & CCATCTCTTTTATCAGGGTTGG & CTCTGTGCAAGTAAGCATCTTACA \\
VEGFC & CCACGGGAGGTGTGTATAGA & CAGGAAGTGTGATTGGCAAA \\
VEGFD & ACTCAGTGCAGCCCTAGAGAA & GAACACGTTCACACAAGGGG \\
OPG & GCTAACCTCACCTTCGAG & TGATTGGACCTGGTTACC \\
RANKL & TTAAGCCAGTGCTTCACGGG & ACGTAGACCACGATGATGTCGC \\
SDF-1 & ATTCTCAACACTCCAAACTGTGC & ACTTTAGCTTCGGGTCAATGC \\
CXCR4 & ACTACACCGAGGAAATGGGCT & CCCACAATGCCAGTTAAGAAGA \\
\hline
\end{tabular}

of tissue were used. Hematoxylin and eosin (H\&E) staining was used to define tumor tissues of the intracardiac injection and orthotopic implantation models. H\&E staining, tartrateresistant acid phosphatase (TRAP) staining (Sigma, USA) and immunohistochemical (IHC) staining were applied on the left tibia tissue of the intratibial injection models. Osteoclast number was assessed as TRAP-positive cells along the tumorbone interface on TRAP-stained sections, and expressed as the osteoblast number per x 200 field. For IHC staining, antigen retrieval was carried out by pepsin antigen retrieval solutions (ZSGB-Bio, China). Primary rabbit anti-Noggin monoclonal antibody (Abcam) and primary rabbit anti-NRP1 monoclonal antibody (ABclonal, USA) were used, respectively. The images were observed under a fluorescence microscope (Leica DM 400B, Germany) and analyzed by Leica Application suite Version 3.8.0 software (Leica, Germany). The IHC result was classified as 0 [equal to negative (-)]; 1-4 [weakly positive $(+)$ ]; 5-8 [moderately positive $(++)$ ] and 9-12 [strongly positive $(+++)$ ]. Two experienced pathologists in a blinded manner identified the scores.

Statistical analysis. Osteoclast data are expressed as the mean \pm SD. Statistical significance was determined by the unpaired Student's t-test. CT score and IHC score were analyzed using Kruskal-Wallis test. Statistical analysis was performed using SPSS 13.0 software. The results with P-values $<0.05$ were considered as statistically significant, and indicated by an asterisk in the figures.

\section{Results}

Construction of the stable CRMP4 gene overexpressing PC3 and DU145 cell lines. After transduction of the CRMP4 ${ }^{+}$ lentivirus of PC3 and DU145 cell lines, FACS was used for a stable overexpressing CRMP4 PC3 and DU145 cell lines. We obtained 30-fold overexpression of CRMP4 at mRNA level by qRT-PCR examination and obvious differential expression at protein level by western blot analysis compared to control PC3 and DU145 cells (Fig. 1).

CRMP4 overexpression shows no difference of bone metastasis capacity in orthotopic implantation and intracardiac injection models. PET/CT scanning of two groups of models showed no obvious bone destruction. In the orthotopic group, PET/CT examination showed abdominal high signal, mandible and abdominal wall high signal were occasionally observed (3/14), but both $\mathrm{CRMP}^{+}$and control groups showed no bone high signal, CT scanning showed skeleton system undamaged (Fig. 2A and C). In the intracardiac injection groups, soft tissue metastasis (subcutaneous and muscle tissue) is the most common metastasis which was $3 / 7$ in the $\mathrm{CRMP}^{+}$group and 7/7 in the control group. Lung metastasis (1/7 CRMP4 ${ }^{+}$group and 5/7 control group) is the second most common metastasis and the other metastasis included liver metastasis (2/7 control group). Some of the animals showed pelvis and spine abnormal signal but no bone destruction in CT scanning and no abnormal bone tissue was identified in anatomy and H\&E staining (Fig. 2B and D).

CRMP4 overexpression inhibits tumor growth in intratibial injection models. High resolution CT scanning of left limb of intratibial injection models showed that both groups developed osteolytic bone destruction. In the CRMP4 ${ }^{+}$ group, three dimensional reconstructive CT imaging showed integrated tibia form and sagittal and coronal view showed an osteoclastic reaction in medullary cavity (Fig. 3A-D). Internal wall of cortical bone decay was common (7/7) and micro-cortical perforation was observed (3/7). Two mice developed fracture but showed no severe bone destruction. In the control group, reconstructive CT images showed 


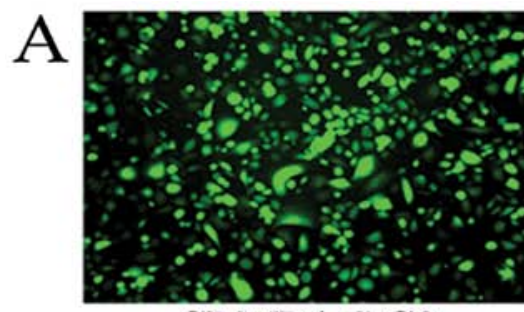

CRMP4+PC3
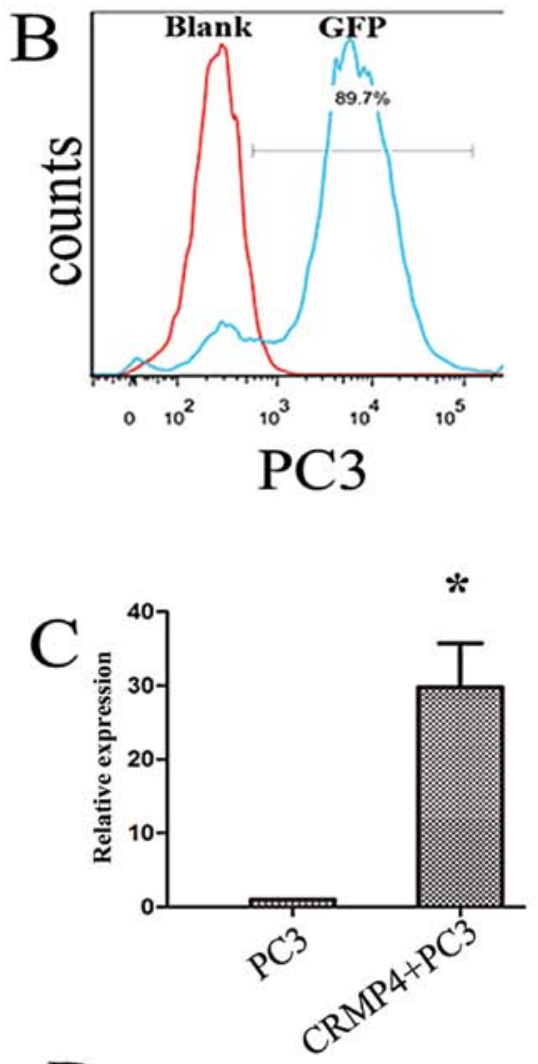

$\mathrm{D}$

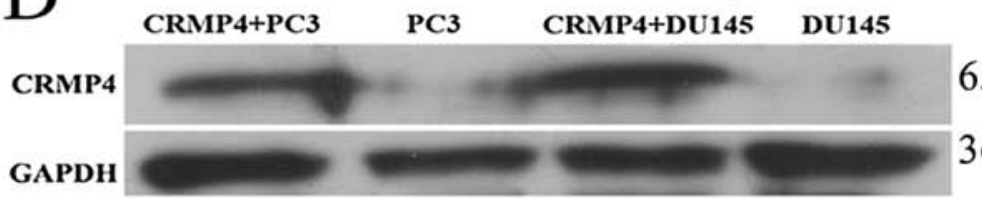

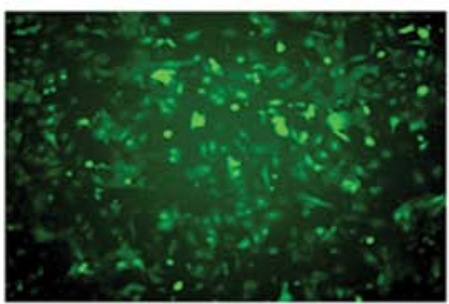

CRMP4+DU145
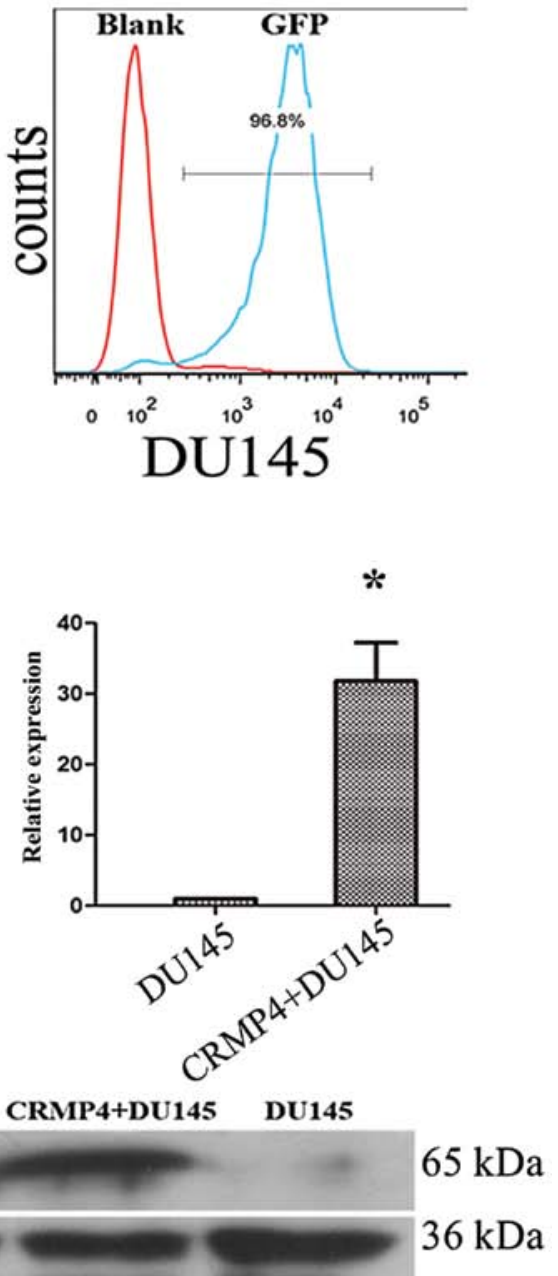

Figure 1. The confirmation of CRMP4 gene upregulation. (A) GFP expression of PC3 and DU145 after CRMP4-GFP lentiviral particles transduction and FACS showed high level GFP expression. (B) FCM analysis of GFP expression of the CRMP4-GFP PC3 and DU145 cell lines. Both group showed high rate of GFP expression. (C) qRT-PCR analysis of CRMP4 gene expression in PC3 and DU145 CRMP4 ${ }^{+}$cell line. Transduction with CRMP4-GFP lentivirus obtained $\sim 30$-fold CRMP4 expression at mRNA level. "P<0.05 vs. control group. (D) CRMP4 protein expression in CRMP4+PC3 and DU145 cell lines. Western blot analysis showed obvious overexpression of CRMP4 protein in CRMP4 lentivirus transfected cell lines.

apparent bone resorption, cortex destruction and fracture was common (4/7) after 40 days of injection (Fig. 3E-H). CT imaging score showed statistical difference $(\mathrm{P}<0.05)$. H\&E staining showed large and irregular tumor cells with a light stain and a big nucleus, compare to a small and round deep stain in normal bone marrow cells. In the CRMP4 ${ }^{+}$group, tumor cell growth was restricted to the marrow cavity. The boundary between cancer cells and bone marrow cells is clear. In the control group, bone cortex destruction is common and in some cases bone marrow cells disappeared completely. Both groups showed cancer cells mixed with smaller bone marrow cells and both group had osteolytic bone destruction (Fig. 5A and B). TRAP staining showed TRAP-positive cells (osteoclast) located at the tumor-bone matrix interface, and no statistical difference of positive cells between the two groups (Figs. 5E and F and 6). IHC staining showed CRMP4 ${ }^{+}$ tumor cells overexpressed Noggin and low expression of NRP1 $(\mathrm{P}<0.05)$, conforming with the in vitro experiments (Fig. 5C, D, G and H).

CRMP4 overexpression enhance Noggin expression in vitro. Noggin/BMP signaling was reported involved in prostate 

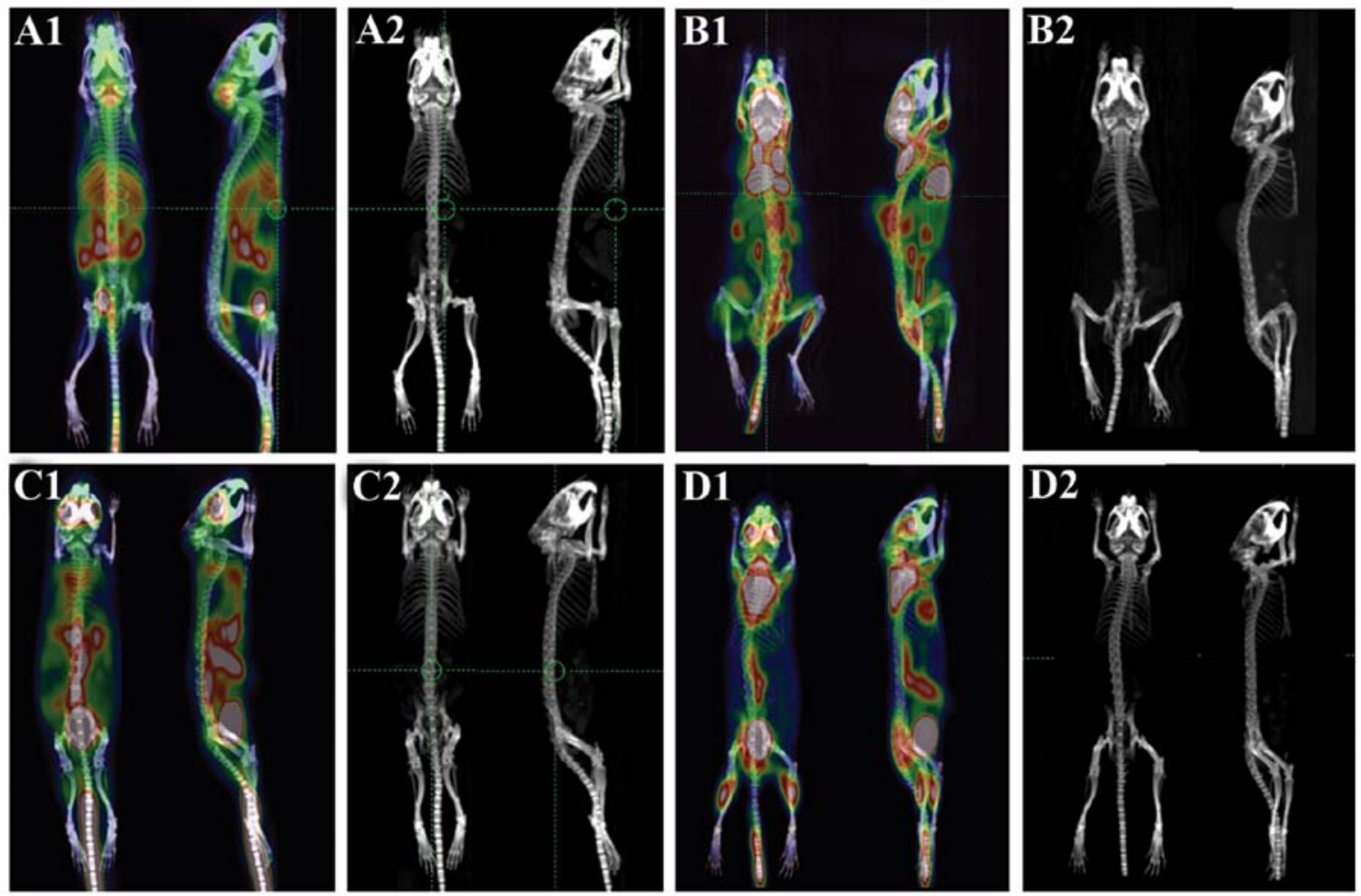

Figure 2. ${ }^{18} \mathrm{~F}-\mathrm{FDG}$ PET/CT examination of the orthotopic implantation and intracardiac injection in the nude mouse model. In the orthotopic implantation group [(A) control group; (C) CRMP4 $4^{+}$group; (A1 and C1) PET/CT scanning; (A2 and C2) CT scanning], PET/CT scanning shows abdominal high signal in both groups. CT scanning showed no bone destruction in the groups. In the intracardiac injection group [(B) control group; (D) CRMP4 ${ }^{+}$group; (B1 and D1) PET/CT scanning; (B2 and D2) CT scanning] PET/CT scanning showed wide soft tissue high signal intensity in control group and CRMP4 ${ }^{+}$group demonstrating less metastasis, neither group showed bone destruction in the CT scan.
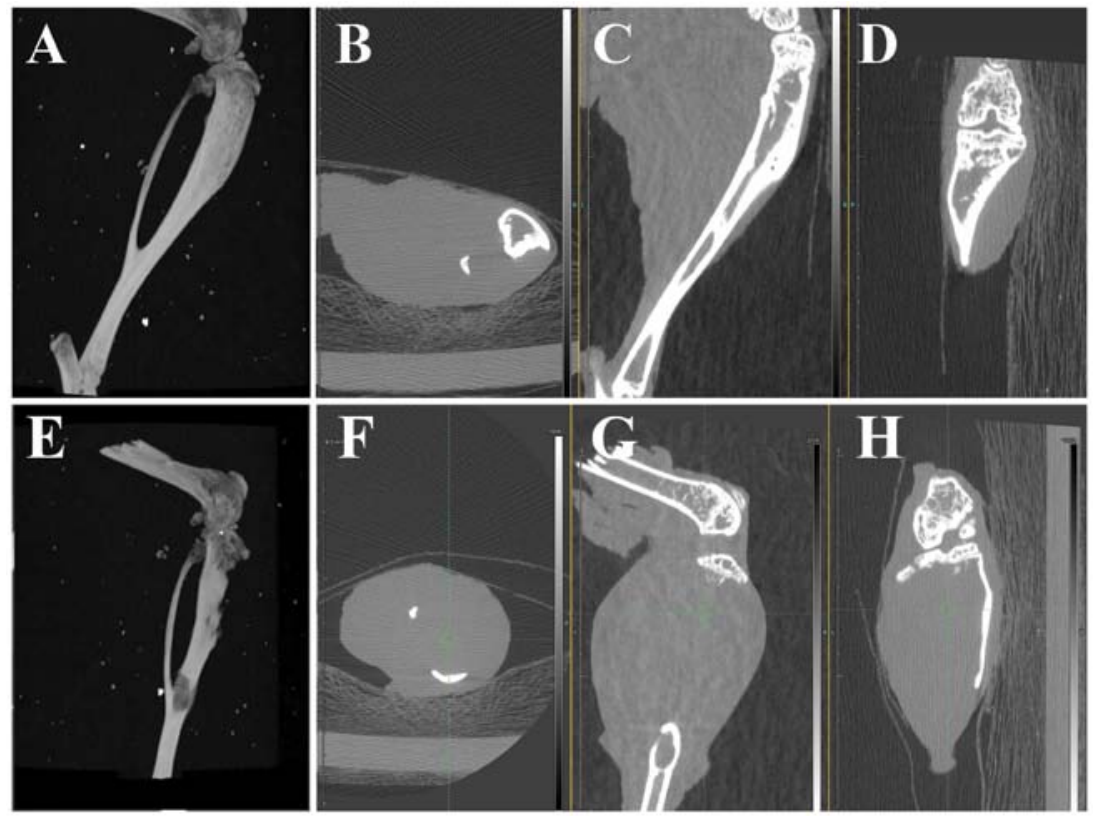

Figure 3. High resolution CT scan of the left limbs of intratibial injection model. (A-D) CRMP4 ${ }^{+}$group; (E-H) control group. Three dimensional reconstruction (A and E) showed no apparent abnormality of CRMP4 ${ }^{+}$group and a severe destruction of tibia of the control group. Transverse view (B and F), sagittal view $(\mathrm{C}$ and $\mathrm{G})$ and coronal view $\left(\mathrm{D}\right.$ and $\mathrm{H}$ ) showed cortical bone intact and mild marrow cavity decay in $\mathrm{CRMP4}{ }^{+}$group and a severe bone and full cortical destruction in the control group. Both groups showed osteolytic bone destruction.

cancer bone metastasis (27), both Noggin/BMP and CRMP play an important role in the developing nervous system (28). We hypothesized CRMP4 combined in the Noggin/
BMP signaling in prostate cancer in the signal pathway. qRT-PCR examination showed both PC3 and DU145 cell line expressed low level of Noggin, BMP2 and BMP4, the cell 


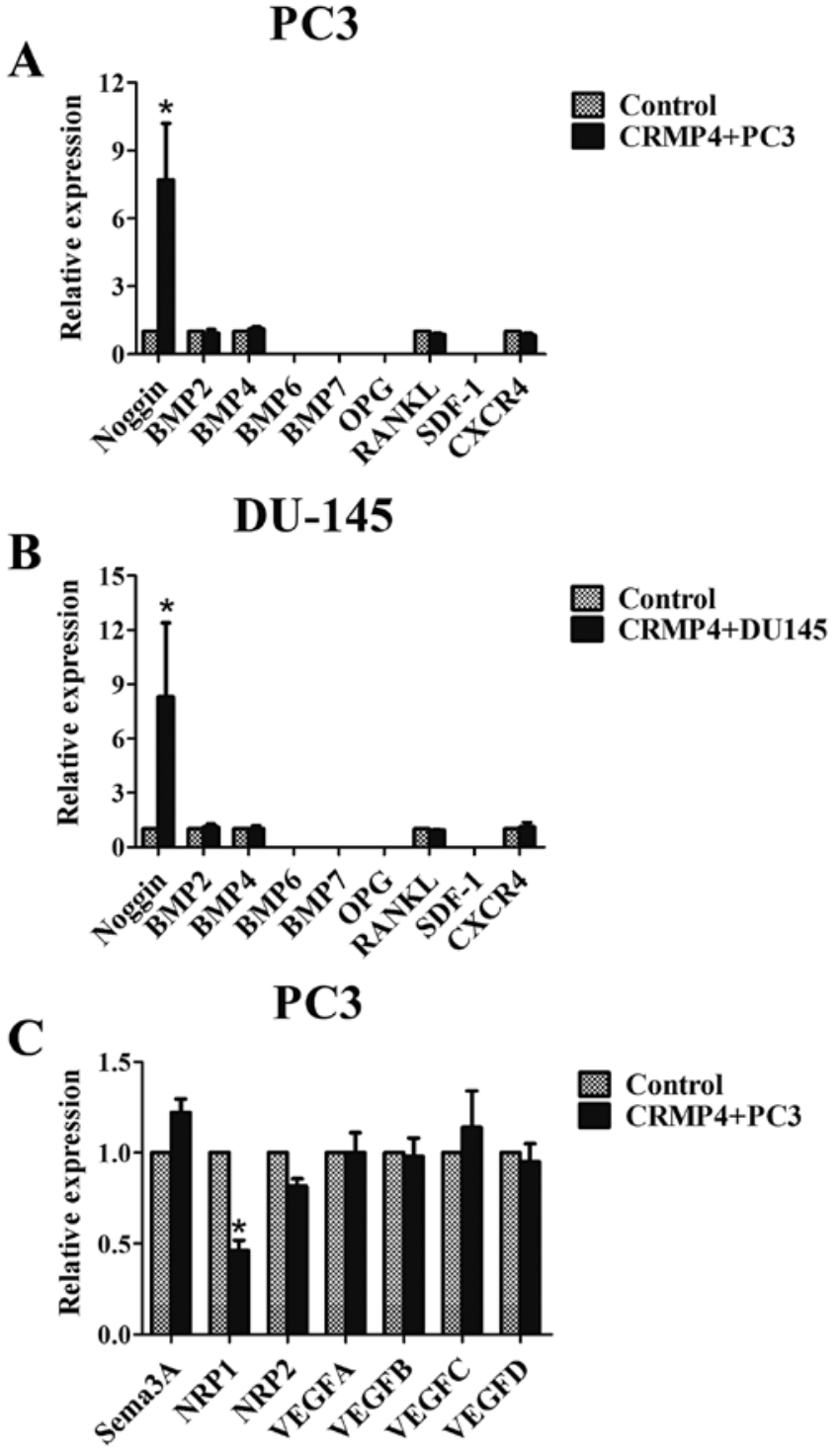

D
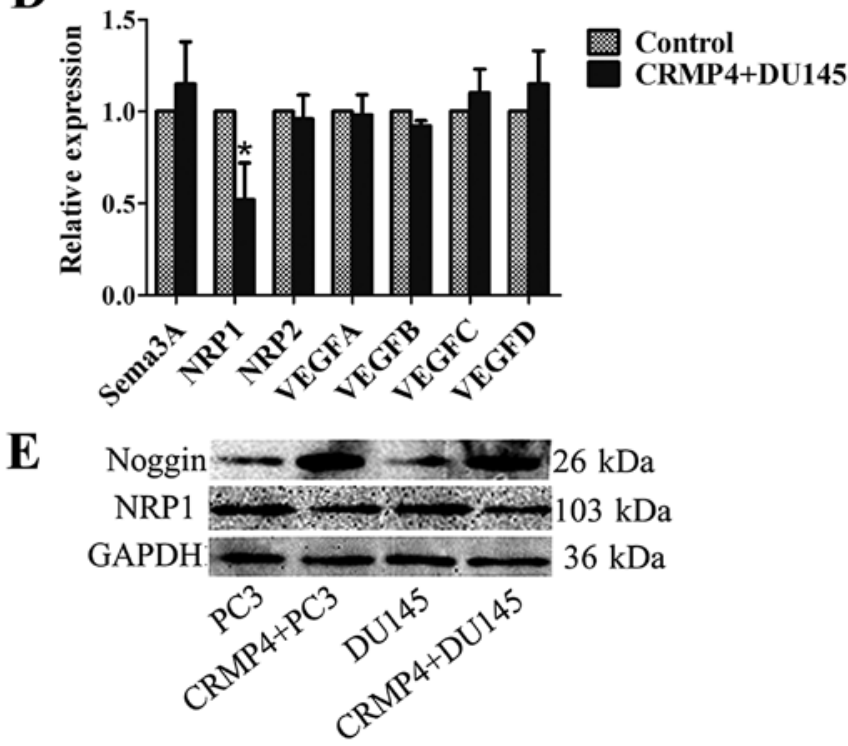

Figure 4. Expression of bone metastasis related cytokines in the CRMP4 ${ }^{+}$ and control cell line. (A-D) qRT-PCR results; (E) western blot analysis of Noggin and NRP1 expression of CRMP4 ${ }^{+}$and control cells. Noggin overexpression and NRP1 downregulation were observed in both PC3 and DU145 CRMP4 ${ }^{+}$cell lines $\left({ }^{*} \mathrm{P}<0.05\right.$ vs. control group). lines expressed no BMP6 and BMP7 (no CT value detected). CRMP4+PC3 and DU145 cell lines overexpress Noggin both in mRNA and protein levels. No significant difference was recorded in the RNA expression of BMP2 and BMP4 in the cell lines (Fig. 4A, B and E).

CRMP4 overexpression inhibits NRP1 expression. CRMP4 is a downstream protein in Sema3A-NRP1 signaling and NRP1 is an important negative regulation transmembranous protein in this signaling (1), NRP1 is a coreceptor for VEGF and promotes VEGFR activation (29,30). We hypothesized that CRMP4 inhibits VEGF combined to its receptor by inhibiting NRP1.qRT-PCR examination showed both PC 3 and DU145 cell lines highly express all four VEGF members and NRP1, with low expression of NRP2 and Sema3A. CRMP4 overexpression reduced NRP1 expression, but had no effect on sema3A, NRP2 and VEGF expression. Western blot examination was confirmed with qRT-PCR examination, NRP1 downregulation was observed in both $\mathrm{CRMP}^{+}$cell lines (Fig. 4C-E).

CRMP4 and other cytokines involved in prostate bone metastasis. SDF-1/CXCR4 pathway is an element in the processes of hematopoietic cell homing to bone during embryonic development (31) and related to cancer bone metastasis $(32,33)$. In our research, we find that both PC3 and DU145 express no SDF-1 gene and low expression of CXCR4 mRNA, and CRMP4 overexpression did not affect CXCR4 mRNA expression. OPG/ RANKL signaling is an important regulator of osteolytic/ osteoblastic balance in bone (34) and it has been shown that OPG and RANKL are key factors mediating osteolytic bone injury in prostate cancer bone metastasis (35). Our research showed both cell lines express OPG and RANKL at a low mRNA lever but these factors are not affected by CRMP4 overexpression (Fig. 4A and B).

\section{Discussion}

Cancer bone metastasis is a complex biological process involved in numerous cell and protein interactions. CRMP is a new cancer related protein, understanding its role in cancer process and the relationship with other cancer related proteins is likely to improve our understanding of cancer physiology and pathology.

In our research, orthotopic implantation and intracardiac injection in nude mouse models showed low rate of bone metastasis. In agreement, previous research showed most immune deficiency mouse implantation models revealed low bone metastatic capability (36). Yang et al (37) reported a prostate orthotopic implantation model by a GFP-PC3 cell line and micrometastasis were identified in the skeleton including the skull, rib, pelvis, femur, and tibia. Corey et al (38) reported a prostate orthotopic implantation model with $\mathrm{PSA}^{+} \mathrm{LuCaP} 23.8$ and LuCaP 35 cells and dectected PSA expression in bone marrow by reverse transcription PCR (RT-PCR) examination, which indicates mircrometastasis formed in the bone. Our experiment showed low skeleton metastasis by ${ }^{18} \mathrm{~F}-\mathrm{FDG}$ probe PET/CT examination in prostate orthotopic implantation $(0 / 14)$ and intracardiac injection models $(0 / 14)$. All the animals formed apparent subcutaneous or abdominal tumors 40 days after implantation but PET/CT showed no obvious 


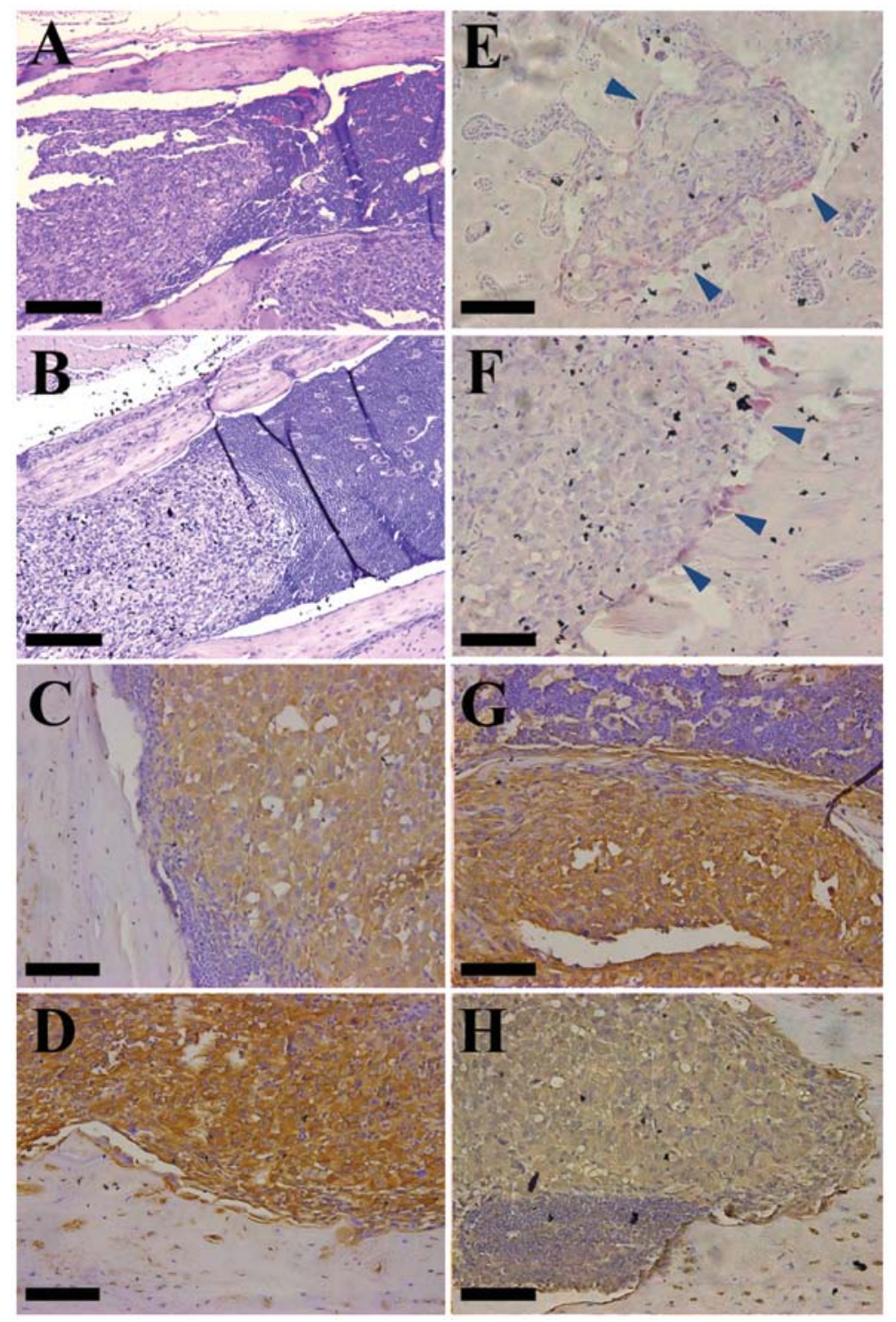

Figure 5. Histological staining of intratibial injection models. (A and B) Bar, $200 \mu \mathrm{m}$; (C-H) bar, $100 \mu \mathrm{m}$. (A, E, C and G) Control group. (B, F, D and H) $\mathrm{CRMP}^{+}$group. (A and B) H\&E staining showed tumor cells are large and lightly stained, compared to small and deep stain in normal bone marrow cells (E and F) TRAP staining showed osteoclast located at the tumor-bone matrix interface. Arrows indicate positive staining. (C and D) IHC staining of Noggin expression, CRMP4 ${ }^{+}$group had significantly higher expression of Noggin than that in the control group. (G and H) IHC staining of NRP1 expression, control group showed high expression of NRP1 of tumor cells.

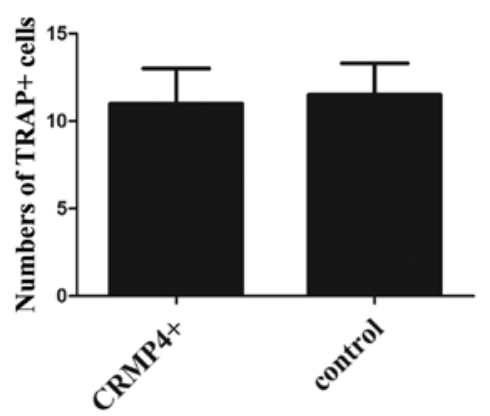

Figure 6. Number of TRAP positive cells, $\mathrm{CRMP}^{+}$and control group, respectively. No statistical difference between the two groups $(\mathrm{P}>0.05$.)

bone abnormal signal and the CT scan showed no bone destruction. This result may imply micrometastatic cancer cell dormancy in a low metabolic rate can not be detected by
${ }^{18} \mathrm{~F}-\mathrm{FDG}$ examination, this result imply tumor cell dormancy in bone mircometastasis and the animal probably die of other metastasis before dormant bone metastatic tumor recur and form a macroscopic metastasis. Intratibial injection model produce stable tumor growth in bone compared to intracardiac injection and orthotopic implantation, this method to some degree is incorrectly referred to as the metastasis model. It failed to reveal the early stage of tumor metastasis, but this method offer a stable condition of tumor cell growth in the bone environment. In the present study, the tumor growth is suppressed by CRMP4 overexpression in bone environment, but whether CRMP4 affects the early stage of prostate cancer bone metastasis need to be further investigated.

Noggin is a bone morphogenetic protein (BMP) antagonist and is an essential regulator of BMP activity (39). Both Noggin-BMP and Sema-CRMP play important roles in the early stage of the nervous system development. Noggin/BMPs 
and the relationship with CRMP were reported in a developing Xenopus nervous system. Kamata et al (40) reported CRMP-2 expression was activated by Noggin and dominant negative BMP receptor in Xenopus embryos. The authors considered that the transcriptional control of XCRMP-2 gene is one of the targets of BMP4 signaling. In prostate cancer, Noggin was demonstrated as a potent protector in bone metastasis $(41,42)$ and BMP family, which include more than a dozen members, demonstrated to have different function in numerous studies $(27,42,43)$. We believe Noggin overexpression may suppress the cell interaction between tumor cells and BMPs in bone matrix, but the specific mechanism is not clear. Based on the reported function of BMP and CRMP in neural development, our results suggest a feedback regulation may exist between the CRMP, Noggin and BMP in prostate cancer.

NRP1 is an important transmembranous co-receptor for VEGF and semaphorin family member. NRP1 acts as a co-receptor for VEGF and promotes VEGFR activation (44), VEGF bind to VEGFR to activate downstream signaling including PI3K, ERK and MAPK pathway, consequently promoting tumor cells proliferation, survival, differentiation, migration and angiogenesis (30). It was shown that Sema3 competes with VEGF for NRP1 binding and prevents angiogenesis $(45,46)$. CRMP4 overexpression and NRP1 downregulation may suppress VEGF in autocrine and paracrine manner by both tumor cells and bone marrow cells, binding to tumor cells VEGFR blocks the downstream pathway. NRP1 downregulation may derive from the activation of Sema3A signaling in tumor cells since most of Sema family proteins are expressed in prostate cancer cells, and further research is needed on this aspect.

OPG/RANKL signaling is a key regulator for osteoclast/ osteoblast balance (34). RANKL binds to its receptor RANK to control osteoclast differentiation, activation and survival. Osteoprotegerin (OPG) blocks ligand binding to RANK, thereby preventing osteoclast differentiation and activation. It has been shown $(47,48)$ that OPG plays protective role in cancer bone metastasis and RANKL promotes osteolytic bone metastasis. In our investigation, CRMP4 overexpression showed no influence on RANKL and OPG expression and the in vivo study showed both groups developed osteolytic bone destruction and no osteoclast cell difference in TRAP staining, indicating CRMP4 overexpression may have no effect on osteoclast/osteoblast balance in bone microenvironment. SDF1/CXCR4 which is reported to promote prostate spread to the bone $(32,33)$, is not affected by CRMP4 overexpression, indicating CRMP4 may not be involved in the early stage of bone metastasis.

In this study, we sought to determine the role of CRMP4 in prostate cancer bone metastasis and the relationship between CRMP4 and cytokines related with bone metastasis. The results from this study demonstrated that CRMP4 inhibits nude mouse tumor growth in a nude intratibial injection model and Noggin and NRP1 may act as downstream cytokines of CRMP4 to mediate the tumor cells in bone environment reaction. We believe other pathways may also exist in CRMP4 regulating NRP1 and Noggin expression in prostate cancer and we will further investigate the downstream biological effects of CRMP4. In conclusion, our research suggested CRMP4 as a potential target for prevention of prostate bone metastasis.

\section{Acknowledgements}

This study was supported by the Natural Science Foundation of China (31170947), the China Postdoctoral Science Foundation (2014M552272), the Guangdong Natural Sciences Foundation of China (S2012020011099). We sincerely thank Tie Niu, Nan Cai, Changchang Jia (Biotherapy Center, The Third Affiliated Hospital of Sun Yat-sen University) for their help in this study.

\section{References}

1. Schmidt EF and Strittmatter SM: The CRMP family of proteins and their role in Sema3A signaling. Adv Exp Med Biol 600: 1-11, 2007.

2. Wang LH and Strittmatter SM: Brain CRMP forms heterotetramers similar to liver dihydropyrimidinase. J Neurochem 69: 2261-2269, 1997.

3. Deo RC, Schmidt EF, Elhabazi A, et al: Structural bases for CRMP function in plexin-dependent semaphorin3A signaling. EMBO J 23: 9-22, 2004

4. Goshima Y, Nakamura F, Strittmatter P, et al: Collapsin-induced growth cone collapse mediated by an intracellular protein related to UNC-33. Nature 376: 509-514, 1995.

5. Fukata Y, Itoh TJ, Kimura T, et al: CRMP-2 binds to tubulin heterodimers to promote microtubule assembly. Nat Cell Biol 4: 583-591, 2002.

6. Gu Y and Ihara Y: Evidence that collapsin response mediator protein-2 is involved in the dynamics of microtubules. J Biol Chem 275: 17917-17920, 2000.

7. Yuasa-Kawada J, Suzuki R, Kano F, et al: Axonal morphogenesis controlled by antagonistic roles of two CRMP subtypes in microtubule organization. Eur J Neurosci 17: 2329-2343, 2003.

8. Arimura N, Inagaki N, Chihara K, et al: Phosphorylation of collapsin response mediator protein-2 by Rho-kinase. Evidence for two separate signaling pathways for growth cone collapse. J Biol Chem 275: 23973-23980, 2000.

9. Kawano Y, Yoshimura T, Tsuboi D, et al: CRMP-2 is involved in kinesin-1-dependent transport of the Sra-1/WAVE1 complex and axon formation. Mol Cell Biol 25: 9920-9935, 2005.

10. Santolini E, Puri C, Salcini AE, et al: Numb is an endocytic protein. J Cell Biol 151: 1345-1352, 2000.

11. Nishimura T, Fukata Y, Kato K, et al: CRMP-2 regulates polarized Numb-mediated endocytosis for axon growth. Nat Cell Biol 5: 819-826, 2003

12. Tan F, Thiele CJ and Li Z: Collapsin response mediator proteins: potential diagnostic and prognostic biomarkers in cancers. Oncol Lett 7: 1333-1340, 2014.

13. Shih JY, Yang SC, Hong TM, et al: Collapsin response mediator protein- 1 and the invasion and metastasis of cancer cells. J Natl Cancer Inst 93: 1392-1400, 2001.

14. Pan SH, Chao YC, Hung PF, et al: The ability of LCRMP-1 to promote cancer invasion by enhancing filopodia formation is antagonized by CRMP-1. J Clin Invest 121: 3189-3205, 2011.

15. Wang WL, Hong TM, Chang YL, et al: Phosphorylation of LCRMP-1 by GSK3beta promotes filopodia formation, migration and invasion abilities in lung cancer cells. PLoS One 7: e31689, 2012.

16. Wu CC, Chen HC, Chen SJ, et al: Identification of collapsing response mediator protein-2 as a potential marker of colorectal carcinoma by comparative analysis of cancer cell secretomes. Proteomics 8: 316-332, 2008.

17. Oliemuller E, Peláez R, Garasa S, et al: Phosphorylated tubulin adaptor protein CRMP-2 as prognostic marker and candidate therapeutic target for NSCLC. Int J Cancer 132: 1986-1995, 2013.

18. Shimada K, Ishikawa T, Nakamura F, et al: Collapsin response mediator protein 2 is involved in regulating breast cancer progression. Breast Cancer: Feb 5, 2013 (Epub ahead of print).

19. Meyronet D, Massoma $\mathrm{P}$, Thivolet F, et al: Extensive expression of collapsin response mediator protein 5 (CRMP5) is a specific marker of high-grade lung neuroendocrine carcinoma. Am J Surg Pathol 32: 1699-1708, 2008.

20. Gao X, Pang J, Li LY, et al: Expression profiling identifies new function of collapsin response mediator protein 4 as a metastasis-suppressor in prostate cancer. Oncogene 29: 4555-4566, 2010.

21. Valastyan S and Weinberg RA: Tumor metastasis: molecular insights and evolving paradigms. Cell 147: 275-292, 2011. 
22. Chambers AF, Groom AC and MacDonald IC: Dissemination and growth of cancer cells in metastatic sites. Nat Rev Cancer 2: 563-572, 2002

23. Nagrath S, Sequist LV, Maheswaran S, et al: Isolation of rare circulating tumour cells in cancer patients by microchip technology. Nature 450: 1235-1239, 2007.

24. Choueiri MB, Tu SM, Yu-Lee LY, et al: The central role of osteoblasts in the metastasis of prostate cancer. Cancer Metastasis Rev 25: 601-609, 2006

25. Chung LW: Prostate carcinoma bone-stroma interaction and its biologic and therapeutic implications. Cancer 97: 772-778, 2003.

26. Wu TT, Sikes RA, Cui Q, et al: Establishing human prostate cancer cell xenografts in bone: induction of osteoblastic reaction by prostate-specific antigen-producing tumors in athymic and SCID/bg mice using LNCaP and lineage-derived metastatic sublines. Int J Cancer 77: 887-894, 1998.

27. Yang S, Zhong C, Frenkel B, et al: Diverse biological effect and smad signaling of bone morphogenetic protein 7 in prostate tumor cells. Cancer Res 65: 5769-5777, 2005.

28. Pera EM, Acosta H, Gouignard N, et al: Active signals, gradient formation and regional specificity in neural induction. Exp Cell Res 321: 25-31, 2014.

29. Ferrara N, Gerber HP and LeCouter J: The biology of VEGF and its receptors. Nat Med 9: 669-676, 2003.

30. Takahashi $\mathrm{H}$ and Shibuya M: The vascular endothelial growth factor (VEGF)/VEGF receptor system and its role under physiological and pathological conditions. Clin Sci 109: 227-241,2005.

31. Hardy CL: The homing of hematopoietic stem cells to the marrow. Am J Med Sci 309: 260-266, 1995.

32. Taichman RS, Cooper C, Keller ET, et al: Use of the stromal cell-derived factor-1/cxcr4 pathway in prostate cancer metastasis to bone. Cancer Res 62: 1832-1837, 2002.

33. Zhang XH, Wang Q, Gerald W, et al: Latent bone metastasis in breast cancer tied to Src-dependent survival signals. Cancer Cell 16: 67-78, 2009.

34. Hsu H, Lacey DL, Dunstan CR, et al: Tumor necrosis factor receptor family member RANK mediates osteoclast differentiation and activation induced by osteoprotegerin ligand. Proc Natl Acad Sci USA 96: 3540-3545, 1999

35. Virk MS, Petrigliano FA and Liu NQ: Influence of simultaneous targeting of the bone morphogenetic protein pathway and RANK-RANKL axis in osteolytic prostate cancer lesion in bone. Bone 44: 160-167, 2009.
36. Singh AS and Figg WD: In vivo models of prostate cancer metastasis to bone. J Urol 174: 820-826, 2005.

37. Yang M, Jiang P and Sun FX: A fluorescent orthotopic bone metastasis model of human prostate cancer. Cancer Res 59 781-786, 1999.

38. Corey E, Quinn JE, Vessella RL, et al: A novel method of generating prostate cancer metastases from orthotopic implants. Prostate 56: 110-114, 2003.

39. Krause C, Guzman A and Knaus P: Noggin. Int J Biochem Cell Biol 43: 478-481, 2011.

40. Kamata T, Daar IO, Subleski M, et al: Xenopus CRMP-2 is an early response gene to neural induction. Brain Res Mol Brain Res 57: 201-210, 1998

41. Feeley BT, Krenek L, Liu N, et al: Overexpression of noggin inhibits BMP-mediated growth of osteolytic prostate cancer lesions. Bone 38: 154-166, 2006.

42. Morrissey C, Brown LG, Pitts TE, et al: Bone morphogenetic protein 7 is expressed in prostate cancer metastases and its effects on prostate tumor cells depend on cell phenotype and the tumor microenvironment. Neoplasia 12: 192-205, 2010.

43. Dai J, Keller J, Zhang J, et al: Bone morphogenetic protein-6 promotes osteoblastic prostate cancer bone metastases through adual mechanism. Cancer Res 65: 8274-8285, 2005.

44. Jackson MW: A potential autocrine role for vascular endothelial growth factor in prostate cancer. Cancer Res 62: 854-859, 2002.

45. Staton CA: Class 3 semaphorins and their receptors in physiological and pathological angiogenesis. Biochem Soc Trans 39: $1565-1570,2011$

46. Miao HQ, Soker S, Feiner L, et al: Neuropilin-1 mediates collapsin-1/semaphorin III inhibition of endothelial cell motility: functional competition of collapsin-1 and vascular endothelial growth factor-165. J Cell Biol 146: 233-242, 1999.

47. Jones DH, Nakashima T, Sanchez OH, et al: Regulation of cancer cell migration and bone metastasis by RANKL. Nature 440 692-696, 2006.

48. Sottnik JL1 and Keller ET: Understanding and targeting osteoclastic activity in prostate cancer bone metastases. Curr Mol Med 13: 626-639, 2013 